\title{
TWO NEW PROOFS OF THE COMPLETE MONOTONICITY OF A FUNCTION INVOLVING THE PSI FUNCTION
}

\author{
Bai-Ni Guo And Feng QI
}

\begin{abstract}
In the present paper, we give two new proofs for the necessary and sufficient condition $\alpha \leq 1$ such that the function $x^{\alpha}[\ln x-\psi(x)]$ is completely monotonic on $(0, \infty)$.
\end{abstract}

\section{Introduction}

Recall 20] that a function $f$ is said to be completely monotonic on an interval $I$ if $f$ has derivatives of all orders on $I$ and

$$
(-1)^{n} f^{(n)}(x) \geq 0
$$

for all $x \in I$ and $n \in \mathbb{N} \cup\{0\}$. The well-known Bernstein's Theorem in [20, p. 160 , Theorem 12a] states that a function $f$ on $[0, \infty)$ is completely monotonic if and only if there exists a bounded and non-decreasing function $\alpha(t)$ such that

$$
f(x)=\int_{0}^{\infty} e^{-x t} \mathrm{~d} \alpha(t)
$$

converges for $x \in[0, \infty)$.

Recall also [4, 5, 8, 16, 18, that a positive function $f$ is said to be logarithmically completely monotonic on an interval $I$ if $f$ has derivatives of all orders on $I$ and

$$
(-1)^{n}[\ln f(x)]^{(n)} \geq 0
$$

for all $x \in I$ and $n \in \mathbb{N}$.

It was proved explicitly in 5, 16, 18 and other articles that a logarithmically completely monotonic function must be completely monotonic. For more information on the logarithmically completely monotonic functions, please refer to [5, 10, 19] and related references therein.

Received October 25, 2008; Revised August 22, 2008.

2000 Mathematics Subject Classification. Primary 33B15, 65R10; Secondary 26A48, $26 \mathrm{~A} 51$. ity.

Key words and phrases. new proof, completely monotonic function, psi function, inequal-

The authors were supported in part by the China Scholarship Council and the Science Foundation of Tianjin Polytechnic University. 
It is well-known that the Euler gamma function is defined by

$$
\Gamma(z)=\int_{0}^{\infty} t^{z-1} e^{-t} \mathrm{~d} t
$$

for $\Re(z)>0$. The logarithmic derivative of $\Gamma(z)$, denoted by $\psi(z)=\frac{\Gamma^{\prime}(z)}{\Gamma(z)}$, is called the psi or digamma function, and $\psi^{(k)}$ for $k \in \mathbb{N}$ are called the polygamma functions.

In [3], the function

$$
\theta(x)=x[\ln x-\psi(x)]
$$

was proved to be decreasing and convex on $(0, \infty)$, with two limits

$$
\lim _{x \rightarrow 0^{+}} \theta(x)=1 \text { and } \lim _{x \rightarrow \infty} \theta(x)=\frac{1}{2}
$$

being presented complicatedly.

In [2, p. 374], it was pointed out that the limits in (6) can follow immediately from the representations

$$
\theta(x)=x \ln x-x \psi(x+1)+1 \quad \text { and } \quad \theta(x)=\frac{1}{2}+\frac{1}{12 x}-\frac{\tau}{120 x^{3}}
$$

for $x>0$ and $\tau \in(0,1)$.

From (6) and the decreasing monotonicity of $\theta(x)$, the inequality

$$
\frac{1}{2 x}<\ln x-\psi(x)<\frac{1}{x}
$$

for $x>0$ is concluded. This extends a result in 13 , which says that the inequality (7) is valid for $x>1$. Refinements and generalizations of $(7)$ were given in [7, 15, 17] and related references therein. For more information, please refer to 14 and related references therein.

In [11, by employing the monotonicity of $\theta(x)$, it was recovered simply that the double inequality

$$
\frac{x^{x-\gamma}}{e^{x-1}}<\Gamma(x)<\frac{x^{x-1 / 2}}{e^{x-1}}
$$

holds for $x>1$, the constants $\gamma$ and $\frac{1}{2}$ are the best possible, the left-hand side inequality in (8) holds also for $0<x<1$, but the right-hand side inequality in (8) reverses, where $\gamma$ is Euler-Mascheroni's constant. Furthermore, by virtue of the decreasing monotonicity and convexity of $\theta(x)$, it was showed in 11 that the function

$$
h(x)=\frac{e^{x} \Gamma(x)}{x^{x-\theta(x)}}
$$

on $(0, \infty)$ has a unique maximum $e$ at $x=1$, with two limits

$$
\lim _{x \rightarrow 0^{+}} h(x)=1 \text { and } \quad \lim _{x \rightarrow \infty} h(x)=\sqrt{2 \pi} .
$$


Consequently, three sharp inequalities

$$
\frac{x^{x-\theta(x)}}{e^{x}}<\Gamma(x) \leq \frac{x^{x-\theta(x)}}{e^{x-1}}
$$

on $(0,1]$,

$$
\frac{\sqrt{2 \pi} x^{x-\theta(x)}}{e^{x}}<\Gamma(x) \leq \frac{x^{x-\theta(x)}}{e^{x-1}}
$$

on $[1, \infty)$, and

$$
I(x, y)<\left\{\frac{x^{\theta(x)} \Gamma(x)}{y^{\theta(y)} \Gamma(y)}\right\}^{1 /(x-y)}
$$

for $x \geq 1$ and $y \geq 1$ with $x \neq y$, where

$$
I(a, b)=\frac{1}{e}\left(\frac{b^{b}}{a^{a}}\right)^{1 /(b-a)}
$$

for $a>0$ and $b>0$ with $a \neq b$ is called the identric or exponential mean, are deduced directly. If $0<x \leq 1$ and $0<y \leq 1$ with $x \neq y$, the inequality (13) is reversed.

In [2, pp. 374-375, Theorem 1], by using the well-known Binet's formula and complicated calculating techniques for integrals, the monotonicity and convexity of $\theta(x)$ was extended to the complete monotonicity: For real number $\alpha$, the function

$$
\theta_{\alpha}(x)=x^{\alpha}[\ln x-\psi(x)]
$$

is completely monotonic on $(0, \infty)$ if and only if $\alpha \leq 1$.

The aim of this paper is to give two new proofs of the complete monotonicity of the function $\theta_{\alpha}(x)$, which can be restated as the following Theorem 1 , since this function $\theta_{\alpha}(x)$ has many meaningful applications as stated above.

Theorem 1. For real number $\alpha$, the function $\theta_{\alpha}(x)$ defined by (15) is completely monotonic on $(0, \infty)$ if and only if $\alpha \leq 1$, with two limits

$$
\lim _{x \rightarrow 0^{+}} \theta_{1}(x)=1, \quad \lim _{x \rightarrow \infty} \theta_{1}(x)=\frac{1}{2}
$$

and, for $\alpha<1$,

$$
\lim _{x \rightarrow 0^{+}} \theta_{\alpha}(x)=\infty, \quad \lim _{x \rightarrow \infty} \theta_{\alpha}(x)=0 .
$$

Remark 1. It is easy to obtain that

$$
\theta_{1}^{\prime}(x)=1+\ln x-\psi(x)-x \psi^{\prime}(x)
$$

and

$$
\theta_{1}^{(k+1)}(x)=\frac{(-1)^{k+1}(k-1) !}{x^{k}}-(k+1) \psi^{(k)}(x)-x \psi^{(k+1)}(x)
$$


for $k \in \mathbb{N}$. From Theorem 1 and the fact that a completely monotonic function which is non-identically zero cannot vanish at any point on $(0, \infty)$ (see $[19$, p. 82]), it is derived that

$$
(-1)^{i} \theta_{1}^{(i)}(x)>0
$$

for $i \in\{0\} \cup \mathbb{N}$ on $(0, \infty)$, which are equivalent to the double inequality (7) and the following inequalities on $(0, \infty)$ :

$$
\begin{gathered}
\psi(x)+x \psi^{\prime}(x)>1+\ln x \\
(-1)^{k+1}\left[(k+1) \psi^{(k)}(x)+x \psi^{(k+1)}(x)\right]<\frac{(k-1) !}{x^{k}}, \quad k \in \mathbb{N} .
\end{gathered}
$$

The inequality (20) may be rewritten as

$$
\psi(x)-\ln x>1-x \psi^{\prime}(x), \quad x \in(0, \infty) .
$$

Substituting the right-hand side inequality in (29) for $k=1$ yields the righthand side inequality in (7). This shows that the inequality (20), equivalently, $(22)$, is better than the right-hand side inequality in (7).

Furthermore, rearranging the inequality (21) and using the right-hand side inequality in (29) lead to

$$
\begin{aligned}
(-1)^{k+1} \psi^{(k)}(x) & <\frac{1}{k+1}\left[\frac{(k-1) !}{x^{k}}+(-1)^{k+2} x \psi^{(k+1)}(x)\right] \\
& <\frac{1}{k+1}\left\{\frac{(k-1) !}{x^{k}}+x\left(\frac{k !}{x^{k+1}}+\frac{(k+1) !}{x^{k+2}}\right)\right\} \\
& =\frac{(k-1) !}{x^{k}}+\frac{k !}{x^{k+1}}, \quad k \in \mathbb{N} .
\end{aligned}
$$

This implies that the inequality $(21)$ is stronger than the right-hand side inequality in (29).

\section{Lemmas}

In order to prove Theorem 1, the following lemmas are needed.

Lemma 1 ([1]). For $i \in \mathbb{N}, x>0, a>0$ and $b>0$,

$$
\begin{aligned}
\psi^{(i-1)}(x+1) & =\psi^{(i-1)}(x)+\frac{(-1)^{i-1}(i-1) !}{x^{i}}, \\
\ln \frac{b}{a} & =\int_{0}^{\infty} \frac{e^{-a t}-e^{-b t}}{t} \mathrm{~d} t \\
\psi^{(i)}(x) & =(-1)^{i+1} \int_{0}^{\infty} \frac{t^{i} e^{-x t}}{1-e^{-t}} \mathrm{~d} t, \\
\psi(x)-\ln x+\frac{1}{x} & =\int_{0}^{\infty}\left(\frac{1}{t}-\frac{1}{e^{t}-1}\right) e^{-x t} \mathrm{~d} t .
\end{aligned}
$$


Lemma $2([17])$. For $x>0$,

$$
\begin{gathered}
\frac{1}{2 x}-\frac{1}{12 x^{2}}<\psi(x+1)-\ln x<\frac{1}{2 x}, \\
\frac{1}{2 x^{2}}-\frac{1}{6 x^{3}}<\frac{1}{x}-\psi^{\prime}(x+1)<\frac{1}{2 x^{2}}-\frac{1}{6 x^{3}}+\frac{1}{30 x^{5}} .
\end{gathered}
$$

Lemma 3. Inequalities

$$
\frac{(k-1) !}{x^{k}}+\frac{k !}{2 x^{k+1}}<(-1)^{k+1} \psi^{(k)}(x)<\frac{(k-1) !}{x^{k}}+\frac{k !}{x^{k+1}}
$$

hold on $(0, \infty)$ for $k \in \mathbb{N}$.

Proof. In [15, Lemma 1.3], the function $\psi(x)-\ln x+\frac{\alpha}{x}$ was proved to be completely monotonic on $(0, \infty)$, i.e.,

$$
(-1)^{i}\left[\psi(x)-\ln x+\frac{\alpha}{x}\right]^{(i)} \geq 0
$$

for $i \geq 0$, if and only if $\alpha \geq 1$, so is its negative, i.e., the inequality (30) is reversed, if and only if $\alpha \leq \frac{1}{2}$. In [6, Theorem 2], the function $\frac{e^{x} \Gamma(x)}{x^{x-\alpha}}$ was proved to be logarithmically completely monotonic on $(0, \infty)$, i.e.,

$$
(-1)^{k}\left[\ln \frac{e^{x} \Gamma(x)}{x^{x-\alpha}}\right]^{(k)} \geq 0
$$

for $k \in \mathbb{N}$, if and only if $\alpha \geq 1$, so is its reciprocal, i.e., the inequality (31) is reversed, if and only if $\alpha \leq \frac{1}{2}$. Considering the fact [19, p. 82] that a completely monotonic function which is non-identically zero cannot vanish at any point on $(0, \infty)$ and rearranging either $(30)$ or $(31)$ leads to the double inequalities $(7)$ and (29). Lemma 3 is proved.

Lemma 4. If $f(x)$ is a function defined in an infinite interval I such that $f(x)-f(x+\varepsilon)>0$ and $\lim _{x \rightarrow \infty} f(x)=\delta$ for $x \in I$ and some $\varepsilon>0$, then $f(x)>\delta$ in $I$.

Proof. By induction, for any $x \in I$,

$$
f(x)>f(x+\varepsilon)>f(x+2 \varepsilon)>\cdots>f(x+k \varepsilon) \rightarrow \delta
$$

as $k \rightarrow \infty$. The proof of Lemma 4 is complete.

Remark 2. Lemma 4 is simple, but it is very effectual in dealing with some problems concerning (logarithmically) completely monotonic properties of functions involving the gamma, psi, polygamma functions. 


\section{The first proof of Theorem 1}

Straightforward computation gives

$$
\begin{aligned}
\theta_{1}(x+1)-\theta_{1}(x) & =(x+1) \ln (x+1)-x \ln x+x[\psi(x)-\psi(x+1)]-\psi(x+1) \\
& =(x+1) \ln (x+1)-x \ln x-\psi(x+1)-1
\end{aligned}
$$

and

$$
\begin{aligned}
{\left[\theta_{1}(x+1)-\theta_{1}(x)\right]^{\prime} } & =\ln (x+1)-\ln x-\psi^{\prime}(x+1) \\
& =\int_{0}^{\infty}\left[\frac{1-e^{-t}}{t}-\frac{t e^{-t}}{1-e^{-t}}\right] e^{-x t} \mathrm{~d} t \\
& =\int_{0}^{\infty} \frac{e^{-t}+e^{t}-t^{2}-2}{t\left(e^{t}-1\right)} e^{-x t} \mathrm{~d} t \\
& >0
\end{aligned}
$$

by using formulas (23), (24) and (25). Hence,

$$
(-1)^{i}\left[\theta_{1}(x+1)-\theta_{1}(x)\right]^{(i)}=\left[(-1)^{i} \theta_{1}^{(i)}(x+1)\right]-\left[(-1)^{i} \theta_{1}^{(i)}(x)\right]<0
$$

on $(0, \infty)$ for $i \in \mathbb{N}$.

Using the inequality (27) yields

$$
\begin{aligned}
(x+1) \ln \left(1+\frac{1}{x}\right)-\frac{1}{2 x}-1 & <\theta_{1}(x+1)-\theta_{1}(x) \\
& <(x+1) \ln \left(1+\frac{1}{x}\right)-\frac{1}{2 x}+\frac{1}{12 x^{2}}-1,
\end{aligned}
$$

which implies that $\lim _{x \rightarrow \infty}\left[\theta_{1}(x+1)-\theta_{1}(x)\right]=0$. Since the function $\theta_{1}(x+$ $1)-\theta_{1}(x)$ is increasing on $(0, \infty)$, it is obtained that $\theta_{1}(x+1)-\theta_{1}(x)<0$ on $(0, \infty)$.

Utilizing (23) and (27) leads easily to $\lim _{x \rightarrow \infty} \theta_{1}(x)=\frac{1}{2}$.

Utilization of (18) and (19) and combination of $(23),(27)$ and (28) yield that $\lim _{x \rightarrow \infty} \theta_{1}^{\prime}(x)=0$. The inequality (29) means that $\lim _{x \rightarrow \infty} \theta_{1}^{(i)}(x)=0$ for $i \geq 2$.

By the above argument and Lemma 4 , it is concluded that $(-1)^{k} \theta_{1}^{(k)}(x) \geq$ 0 on $(0, \infty)$ for $k \geq 0$, which means that the function $\theta_{1}(x)$ is completely monotonic on $(0, \infty)$ with $\lim _{x \rightarrow \infty} \theta_{1}(x)=\frac{1}{2}$.

The validity of the $\operatorname{limit}_{\lim _{x \rightarrow 0^{+}}} \theta_{1}(x)=1$ follows from the formula (26).

It is clear that $\theta_{\alpha}(x)=x^{\alpha-1} \theta_{1}(x)$ and $x^{\alpha-1}$ is also completely monotonic on $(0, \infty)$ for $\alpha<1$. Since the product of any finite completely monotonic functions on an interval $I$ is also completely monotonic on $I$, the function $\theta_{\alpha}(x)$ is completely monotonic on $(0, \infty)$ for $\alpha<1$.

Conversely, if the function $\theta_{\alpha}(x)$ is completely monotonic on $(0, \infty)$, then $\theta_{\alpha}(x)$ is decreasing and positive on $(0, \infty)$. From the formula $(23)$ and the 
inequality (27), it follows that

$$
\frac{1}{2 x}+\frac{1}{12 x^{2}}>\ln x-\psi(x)>\frac{1}{2 x}
$$

and

$$
\frac{1}{2 x^{1-\alpha}}+\frac{1}{12 x^{2-\alpha}}>x^{\alpha}[\ln x-\psi(x)]>\frac{1}{2 x^{1-\alpha}}
$$

for $x>0$, which means that $x^{\alpha}[\ln x-\psi(x)]$ tends to $\infty$ as $x \rightarrow \infty$ if $\alpha>1$. This contradicts with the decreasingly monotonic property of $\theta_{\alpha}(x)$ on $(0, \infty)$. Hence, the necessary condition $\alpha \leq 1$ follows.

It is obvious that the inequality (33) implies the two limits in (17). The proof of Theorem 1 is complete.

\section{The second proof of Theorem 1}

Let

$$
h(t)=\frac{1}{t}-\frac{1}{e^{t}-1}=\frac{e^{t}-1-t}{t\left(e^{t}-1\right)}
$$

for $t \neq 0$ and $h(0)=\frac{1}{2}$. Integration by part in (26) yields

$$
\begin{aligned}
\psi(x)-\ln x+\frac{1}{x} & =-\frac{1}{x}\left\{\left.\left[h(t) e^{-x t}\right]\right|_{t=0} ^{t=\infty}-\int_{0}^{\infty} h^{\prime}(t) e^{-x t} \mathrm{~d} t\right\} \\
& =\frac{1}{2 x}+\frac{1}{x} \int_{0}^{\infty} h^{\prime}(t) e^{-x t} \mathrm{~d} t .
\end{aligned}
$$

Multiplying on all sides of (35) by $x$ and rearranging gives

$$
x[\ln x-\psi(x)]=\frac{1}{2}-\int_{0}^{\infty} h^{\prime}(t) e^{-x t} \mathrm{~d} t .
$$

In [9, 12, 21] and related references therein, the function $h(t)$ was shown to be decreasing on $(-\infty, \infty)$, concave on $(-\infty, 0)$ and convex on $(0, \infty)$. This means that the function $\theta_{1}(x)$ is completely monotonic on $(0, \infty)$ and that the second limit in (16) follows. This means that if $\alpha>1$, then the function $\theta_{\alpha}(x)=x^{\alpha-1} \theta_{1}(x)$ tends to infinity for $x$ tending to infinity and therefore it cannot be completely monotonic, that is, the condition $\alpha \leq 1$ is necessary. The second proof of Theorem 1 is complete.

Remark 3 . The second proof of Theorem 1 can also be demonstrated as follows. It is easy to see that

$$
\frac{1}{x}=\int_{0}^{\infty} e^{-x u} \mathrm{~d} u, \quad x>0 .
$$

Substituting it into $(26)$ gives

$$
\ln x-\psi(x)=\int_{0}^{\infty}\left(\frac{1}{1-e^{-t}}-\frac{1}{t}\right) e^{-x t} \mathrm{~d} t \triangleq \int_{0}^{\infty} \rho(t) e^{-x t} \mathrm{~d} t .
$$


An integration by part and a multiplication by $x$ yield

$$
x[\ln x-\psi(x)]=\frac{1}{2}+\int_{0}^{\infty} \rho^{\prime}(t) e^{-x t} \mathrm{~d} t,
$$

where

$$
\rho^{\prime}(t)=\frac{1}{t^{2}}-\frac{e^{-t}}{\left(1-e^{-t}\right)^{2}}=\frac{2 e^{-t}}{t^{2}\left(1-e^{-t}\right)^{2}}\left(\frac{e^{t}+e^{-t}}{2}-1-\frac{t^{2}}{2}\right) .
$$

Making use of the power series expansion of $e^{t}$ at $t=0$ reveals easily that $\rho^{\prime}(t)$ is positive on $(0, \infty)$. So the function $\theta_{1}(x)$ is completely monotonic with the limit $\frac{1}{2}$ at infinity.

Acknowledgments. The authors appreciate the anonymous referees for their helpful comments on and valuable suggestions to the original version of this manuscript.

\section{References}

[1] M. Abramowitz and I. A. Stegun (Eds), Handbook of mathematical functions with formulas, graphs, and mathematical tables, National Bureau of Standards Applied Mathematics Series, 55 Superintendent of Documents, U.S. Government Printing Office, Washington, D.C. 1965.

[2] H. Alzer, On some inequalities for the gamma and psi functions, Math. Comp. 66 (1997), no. 217, 373-389.

[3] G. D. Anderson, R. W. Barnard, K. C. Richards, M. K. Vamanamurthy, and M. Vuorinen, Inequalities for zero-balanced hypergeometric functions, Trans. Amer. Math. Soc. 347 (1995), no. 5, 1713-1723.

[4] R. D. Atanassov and U. V. Tsoukrovski, Some properties of a class of logarithmically completely monotonic functions, C. R. Acad. Bulgare Sci. 41 (1988), no. 2, 21-23.

[5] C. Berg, Integral representation of some functions related to the gamma function, Mediterr. J. Math. 1 (2004), no. 4, 433-439.

[6] Ch.-P. Chen and F. Qi, Logarithmically completely monotonic functions relating to the gamma function, J. Math. Anal. Appl. 321 (2006), no. 1, 405-411.

[7] L. Gordon, A stochastic approach to the gamma function, Amer. Math. Monthly 101 (1994), no. 9, 858-865.

[8] A. Z. Grinshpan and M. E. H. Ismail, Completely monotonic functions involving the gamma and q-gamma functions, Proc. Amer. Math. Soc. 134 (2006), no. 4, 1153-1160.

[9] B.-N. Guo, A.-Q. Liu, and F. Qi, Monotonicity and logarithmic convexity of three functions involving exponential function, J. Korea Soc. Math. Educ. Ser. B Pure Appl. Math. 15 (2008), no. 4, 387-392.

[10] B.-N. Guo and F. Qi, Some logarithmically completely monotonic functions related to the gamma function, J. Korean Math. Soc. 47 (2010), to appear.

[11] B.-N. Guo, Y.-J. Zhang, and F. Qi, Refinements and sharpenings of some double inequalities for bounding the gamma function, J. Inequal. Pure Appl. Math. 9 (2008), no. 1, Art. 17, 5 pages; Available online at http://jipam.vu.edu.au/article.php?sid=953

[12] A.-Q. Liu, G.-F. Li, B.-N. Guo, and F. Qi, Monotonicity and logarithmic concavity of two functions involving exponential function, Internat. J. Math. Ed. Sci. Tech. 39 (2008), no. 5, 686-691.

[13] H. Minc and L. Sathre, Some inequalities involving $(r !)^{1 / r}$, Proc. Edinburgh Math. Soc. (2) $14(1964 / 1965), 41-46$. 
[14] F. Qi, Bounds for the ratio of two gamma functions, RGMIA Res. Rep. Coll. 11 (2008), no. 3, Art. 1; Available online at http://www.staff.vu.edu.au/rgmia/v11n3.asp

[15] _ Three classes of logarithmically completely monotonic functions involving gamma and psi functions, Integral Transforms Spec. Funct. 18 (2007), no. 7, 503-509.

[16] F. Qi and Ch.-P. Chen, A complete monotonicity property of the gamma function, J. Math. Anal. Appl. 296 (2004), no. 2, 603-607.

[17] F. Qi, R.-Q. Cui, Ch.-P. Chen, and B.-N. Guo, Some completely monotonic functions involving polygamma functions and an application, J. Math. Anal. Appl. 310 (2005), no. 1, 303-308.

[18] F. Qi and B.-N. Guo, Complete monotonicities of functions involving the gamma and digamma functions, RGMIA Res. Rep. Coll. 7 (2004), no. 1, Art. 8, 63-72.

[19] F. Qi, B.-N. Guo, and Ch.-P. Chen, Some completely monotonic functions involving the gamma and polygamma functions, J. Aust. Math. Soc. 80 (2006), no. 1, 81-88.

[20] D. V. Widder, The Laplace Transform, Princeton University Press, Princeton, 1941.

[21] Sh.-Q. Zhang, B.-N. Guo, and F. Qi, A concise proof for properties of three functions involving the exponential function, Appl. Math. E-Notes 9 (2009), 177-183.

BAI-Ni GuO

SCHOOL OF MATHEMATICS AND INFORMATICS

Henan Polytechnic University

Jiaozuo City, Henan Province, 454010, P. R. China

E-mail address: bai.ni.guo@gmail.com, bai.ni.guo@hotmail.com

FENG QI

Department of Mathematics

College of Science

Tianjin Polytechnic University

Tianjin City, 300160, P. R. China

E-mail address: qifeng618@gmail.com, qifeng618@hotmail.com, qifeng618@qq.com 